\title{
SMALL ENERGY SCATTERING FOR THE ZAKHAROV SYSTEM WITH RADIAL SYMMETRY
}

\author{
ZIHUA GUO ${ }^{1,2}$, KENJI NAKANISHI ${ }^{3}$
}

\begin{abstract}
We prove small energy scattering for the 3D Zakharov system with radial symmetry. The main ingredients are normal form reduction and the radialimproved Strichartz estimates.
\end{abstract}

\section{InTRODUCTION}

In this paper, we consider the Cauchy problem for the 3D Zakharov system

$$
\left\{\begin{array}{l}
i \dot{u}-\Delta u=n u, \\
\ddot{n} / \alpha^{2}-\Delta n=-\Delta|u|^{2},
\end{array}\right.
$$

with the initial data

$$
u(0, x)=u_{0}, n(0, x)=n_{0}, \dot{n}(0, x)=n_{1},
$$

where $(u, n)(t, x): \mathbb{R}^{1+3} \rightarrow \mathbb{C} \times \mathbb{R}$, and $\alpha>0$ denotes the ion sound speed. It preserves $\|u(t)\|_{L_{x}^{2}}$ and the energy

$$
E=\int_{\mathbb{R}^{3}}|\nabla u|^{2}+\frac{\left|D^{-1} \dot{n}\right|^{2} / \alpha^{2}+|n|^{2}}{2}-n|u|^{2} d x,
$$

where $D:=\sqrt{-\Delta}$, as well as the radial symmetry

$$
(u, n)(t, x)=(u, n)(t,|x|) .
$$

We consider those solutions with such symmetry and finite energy, hence

$$
\left(u_{0}, n_{0}, n_{1}\right) \in H_{r}^{1}\left(\mathbb{R}^{3}\right) \times L_{r}^{2}\left(\mathbb{R}^{3}\right) \times \dot{H}_{r}^{-1}\left(\mathbb{R}^{3}\right) .
$$

We are interested in the scattering for small data in the above function space, as it will open the possibility to discuss about global dynamics of general large solutions (under the radial symmetry).

This system (1.1) was introduced by Zakharov [18 as a mathematical model for the Langmuir turbulence in unmagnetized ionized plasma. It has been extensively studied. Local wellposedness (without symmetry) is well known. For example, the well-posedness in the energy space was proved in [3] for $d=2,3$ and in [5] for $d=1$. The improvement was obtained to the critical regularity in [5, 2] for $d=1,2$, and to the full subcritical regularity in [5, 1] for $d \geq 4, d=3$. The well-posedness for the system on the torus was studied in [17, 8]. These results all follow from the iteration argument by using Bourgain's $X^{s, b}$ space. For the subsonic limit to NLS (as $\alpha \rightarrow \infty$ ), uniform local wellposedness and convergence to a NLS solution has been obtained in [14, 11, 7, 9]. Concerning the long time and blow-up behavior, Merle [10] obtained blow-up in finite or infinite time for negative energy, and the 
scattering theory was studied in [12, 16, 4] for prescribed asymptotic states (namely the final data problem) in weighted Sobolev spaces.

The main difficulties for the scattering in the Sobolev space are derivative loss and slow dispersion of the wave equation together with the quadratic nonlinearity. Our idea is to combine the normal form technique, which was first used in a dispersive PDE context by Shatah [15], and the improved Strichartz estimate for radial functions that was obtained recently in [6]. The normal form transform was used in [13] for the Klein-Gordon-Zakharov system (that is the system where the Schrödinger equation is replaced with the Klein-Gordon) and they got the scattering from initial data small in weighted Sobolev spaces without symmetry. Their estimates rely on Klainerman's vector field method, which is not directly applicable to the standard Zakharov system (1.1) because of the mixed linear part.

Theorem 1.1. If $\left(u_{0}, n_{0}, n_{1}\right)$ are all radial and is small enough in the norm of (1.5), then the solution $(u, n)$ scatters in this space as $t \rightarrow \pm \infty$. More precisely, there are unique solutions $\left(u^{ \pm}, n^{ \pm}\right)$of $0=i \dot{u}^{ \pm}-\Delta u^{ \pm}=\ddot{n}^{ \pm} / \alpha^{2}-\Delta n^{ \pm}$satisfying

$$
\lim _{t \rightarrow \pm \infty}\left[\left\|u(t)-u^{ \pm}(t)\right\|_{H^{1}}+\left\|n(t)-n^{ \pm}(t)\right\|_{L^{2}}+\left\|\dot{n}(t)-\dot{n}^{ \pm}(t)\right\|_{\dot{H}^{-1}}\right]=0 .
$$

Remark 1. Global well-posedness (without symmetry) with small norm in the energy space was proved in [3]. The novelty of Theorem 1.1] is that the solution with radial symmetry also scatters. Our proof is much simpler than those analysis [12, 16, 4, 13. in weighted Sobolev spaces.

\section{TRANSFORM OF EQUATION}

This section is devoted to transform the equation by using the normal form. It is convenient first to change the system into first order as usual. Let

$$
N:=n-i D^{-1} \dot{n} / \alpha,
$$

then $n=\operatorname{Re} N=(N+\bar{N}) / 2$ and the equations for $(u, N)$ are

$$
\left\{\begin{array}{l}
\left(i \partial_{t}-\Delta\right) u=N u / 2+\bar{N} u / 2 \\
\left(i \partial_{t}+\alpha D\right) N=\alpha D|u|^{2}
\end{array}\right.
$$

One can easily see that

$$
\|n\|_{L^{2}}^{2}+\left\|D^{-1} \dot{n}\right\|_{L^{2}}^{2} / \alpha^{2}=\|N\|_{L^{2}}^{2},
$$

and a free wave $n(t)$ is transformed into a free wave $N(t)=e^{i t \alpha D} N(0)$. In our proof, the term $\bar{N} u$ makes no essential difference from $N u$, and hence for simplicity, we assume the nonlinear term in first equation of (2.2) is $N u$.

Now we introduce some notations. We use $S(t), W_{\alpha}(t)$ to denote the Schrödinger, wave semigroups:

$$
S(t) \phi=\mathcal{F}^{-1} e^{i t|\xi|^{2}} \hat{\phi}, \quad W_{\alpha}(t) \phi=\mathcal{F}^{-1} e^{i \alpha t|\xi|} \hat{\phi}, \quad \hat{\phi}=\mathcal{F} \phi .
$$

Let $\eta_{0}: \mathbb{R} \rightarrow[0,1]$ denote an radial smooth function supported in $\{|\xi| \leq 8 / 5\}$ and equal to 1 in $\{|\xi| \leq 5 / 4\}$. For $k \in \mathbb{Z}$ let $\chi_{k}(\xi)=\eta_{0}\left(\xi / 2^{k}\right)-\eta_{0}\left(\xi / 2^{k-1}\right)$ and $\chi_{\leq k}(\xi)=\eta_{0}\left(\xi / 2^{k}\right)$. For $k \in \mathbb{Z}$ let $P_{k}, P_{\leq k}$ denote the operators on $L^{2}\left(\mathbb{R}^{3}\right)$ defined by $\widehat{P_{k} u}(\xi)=\chi_{k}(|\xi|) \widehat{u}(\xi), \widehat{P_{\leq k} u}(\xi)=\chi_{\leq k}(|\xi|) \widehat{u}(\xi)$. 
For any pair of functions $u$ and $v$, we use $(u v)_{L H},(u v)_{H H},(u v)_{H L}$ to denote the three different interactions

$$
(u v)_{L H}:=\sum_{k \in \mathbb{Z}} P_{\leq k-5} u P_{k} v,(u v)_{H L}:=\sum_{k \in \mathbb{Z}} P_{k} u P_{\leq k-5} v,(u v)_{H H}:=\sum_{\substack{\left|k_{1}-k_{2}\right| \leq 4 \\ k_{1}, k_{2} \in \mathbb{Z}}} P_{k_{1}} u P_{k_{2}} v .
$$

To distinguish the resonant interaction, we also use

$$
(u v)_{\alpha L}=\sum_{\substack{\left|k-\log _{2} \alpha\right| \leq 1, k \in \mathbb{Z}}} P_{k} u P_{\leq k-5} v,(u v)_{X L}=\sum_{\substack{k-\log _{2} \alpha \mid>1 \\ k \in \mathbb{Z}}} P_{k} u P_{\leq k-5} v
$$

and similarly $(u v)_{L \alpha},(u v)_{L X}$. It is obvious that we have

$$
\begin{aligned}
u v & =(u v)_{H H}+(u v)_{L H}+(u v)_{H L} \\
& =(u v)_{H H}+(u v)_{L \alpha}+(u v)_{L X}+(u v)_{\alpha L}+(u v)_{X L},
\end{aligned}
$$

and they are all radial if $u, v$ are both radial. All these expressions should be understood as bilinear operators acting on $u$ and $v$, rather than operators on the product $u v$. More explicitly, for any such index $*=H H, H L, \alpha L$, etc., we denote the bilinear symbol (multiplier) by

$$
\mathcal{F}(u v)_{*}=\int \mathcal{P}_{*} \hat{u}(\xi-\eta) \hat{v}(\eta) d \eta
$$

Finite sum of those bilinear operators are denoted by the sum of indices:

$$
(u v)_{*_{1}+*_{2}+\cdots}=(u v)_{*_{1}}+(u v)_{*_{2}}+\cdots
$$

From Duhamel's formula and taking a Fourier transform, we get that the first equation of (2.2) is equivalent to

$$
\begin{aligned}
\hat{u} & =e^{i t|\xi|^{2}} \hat{u}_{0}-i \int_{0}^{t} e^{i(t-s)|\xi|^{2}} \mathcal{F}(N u)_{X L} d s-i \int_{0}^{t} e^{i(t-s)|\xi|^{2}} \mathcal{F}(N u)_{H H+L H+\alpha L} d s \\
& :=I+I I+I I I .
\end{aligned}
$$

Using the equation (2.2) again, we get that

$$
\begin{aligned}
\partial_{t}\left(e^{-i t|\xi|^{2}} \hat{u}\right) & =-i e^{-i t|\xi|^{2}}(\hat{N} * \hat{u})(\xi), \\
\partial_{t}\left(e^{-i \alpha t|\xi|} \hat{N}\right) & =-i e^{-i \alpha t|\xi|} \alpha|\xi|(\hat{u} * \hat{\bar{u}})(\xi) .
\end{aligned}
$$

Thus we have

$$
\begin{aligned}
I I & =-i \int_{0}^{t} e^{i(t-s)|\xi|^{2}} \mathcal{P}_{X L} \hat{N}(s, \xi-\eta) \hat{u}(s, \eta) d \eta d s \\
& =-i e^{i t|\xi|^{2}} \int_{0}^{t} \int \mathcal{P}_{X L} e^{i s \omega}\left[e^{-i \alpha s|\xi-\eta|} \hat{N}(s, \xi-\eta)\right]\left[e^{-i s|\eta|^{2}} \hat{u}(s, \eta)\right] d \eta d s
\end{aligned}
$$

where the resonance function

$$
\omega:=-|\xi|^{2}+\alpha|\xi-\eta|+|\eta|^{2}
$$

in the support of $\mathcal{P}_{X L}: \alpha \nsim|\xi-\eta| \sim|\xi| \gg|\eta|$, has the following size

$$
|\omega| \sim|\xi|^{2}+\alpha|\xi|
$$


This means roughly that for the non-resonant interactions in $\mathcal{P}_{X L}$, we gain $|\xi|^{-2}$ for high frequencies $(|\xi|>1)$, and lose $|\xi|^{-1}$ for low frequencies $(|\xi|<1)$, by the time integration. The gain for high frequencies has been used for the local wellposedness in the $X^{s, b}$ spaces. In general, the lower frequencies can be more problematic in the scattering problems, but it will turn out that we can absorb $|\xi|^{-1}$ by the Sobolev embedding.

Thus from integration by parts, we get

$$
\begin{aligned}
I I= & -e^{i t|\xi|^{2}} \int_{0}^{t} \int \mathcal{P}_{X L} \omega^{-1} \partial_{s}\left(e^{i s \omega}\right) e^{-i \alpha s|\xi-\eta|} \hat{N}(s, \xi-\eta) e^{-i s|\eta|^{2}} \hat{u}(s, \eta) d \eta d s \\
= & -\int \mathcal{P}_{X L} \omega^{-1}\left[\hat{N}(t, \xi-\eta) \hat{u}(t, \eta)-e^{i t|\xi|^{2}} \hat{N}(0, \xi-\eta) \hat{u}(0, \eta)\right] d \eta \\
& -\alpha \int_{0}^{t} \int \mathcal{P}_{X L} \omega^{-1} e^{i(t-s)|\xi|^{2}}|\xi-\eta| \widehat{|u|^{2}}(\xi-\eta) \hat{u}(s, \eta) d \eta d s \\
& -\frac{1}{2} \int_{0}^{t} \int e^{i(t-s)|\xi|^{2}} \mathcal{P}_{X L} \omega^{-1} \hat{N}(s, \xi-\eta)(\hat{N} * \hat{u})(\eta) d \eta d s .
\end{aligned}
$$

We introduce a bilinear Fourier multiplier in the form

$$
\Omega(f, g)=\mathcal{F}^{-1} \int \mathcal{P}_{X L} \omega^{-1} \hat{f}(\xi-\eta) \hat{g}(\eta) d \eta
$$

Then we have

$$
\begin{aligned}
I I= & -\mathcal{F} \Omega(N, u)(t)+e^{i t|\xi|^{2}} \mathcal{F} \Omega(N, u)(0) \\
& -i \alpha \int_{0}^{t} e^{i(t-s)|\xi|^{2}} \mathcal{F} \Omega\left(D|u|^{2}, u\right)(s) d s-\frac{i}{2} \int_{0}^{t} e^{i(t-s)|\xi|^{2}} \mathcal{F} \Omega(N, N u)(s) d s .
\end{aligned}
$$

Thus we obtain

$$
\begin{aligned}
u= & S(t) u_{0}+S(t) \Omega(N, u)(0)-\Omega(N, u)(t)-i \alpha \int_{0}^{t} S(t-s) \Omega\left(D|u|^{2}, u\right)(s) d s \\
& -\frac{i}{2} \int_{0}^{t} S(t-s) \Omega(N, N u)(s) d s-i \int_{0}^{t} S(t-s)(N u)_{L H+H H+\alpha L} d s .
\end{aligned}
$$

For the second equation in (2.2), similarly, we can apply the normal form reduction for the high-low interaction, and then get that it is equivalent to

$$
\begin{aligned}
N= & W_{\alpha}(t) N_{0}+W_{\alpha}(t) D \tilde{\Omega}(u, u)(0)-D \tilde{\Omega}(u, u)(t)-\int_{0}^{t} W_{\alpha}(t-s) D(u \bar{u})_{H H+\alpha L+L \alpha} d s \\
& -\int_{0}^{t} W_{\alpha}(t-s)(D \tilde{\Omega}(N u, u)+D \tilde{\Omega}(u, N u))(s) d s
\end{aligned}
$$

where $\tilde{\Omega}$ is a bilinear Fourier multiplier in the form

$$
\tilde{\Omega}(f, g)=\mathcal{F}^{-1} \int \mathcal{P}_{X L+L X} \frac{\hat{f}(\xi-\eta) \hat{\bar{g}}(\eta)}{|\xi-\eta|^{2}-|\eta|^{2}-\alpha|\xi|} d \eta .
$$


It's easy to see that $\tilde{\Omega}$ behaves "essentially" the same as $\Omega$. The transformed equations look "roughly" (neglecting the difference between $N$ and $\bar{N}$ ),

$$
\begin{aligned}
& \left(i \partial_{t}+D^{2}\right)(u-\Omega(N, u))=(N u)_{L H+H H+\alpha L}+\Omega\left(D|u|^{2}, u\right)+\Omega(N, N u), \\
& \left(i \partial_{t}+\alpha D\right)(N-D \tilde{\Omega}(u, u))=D(u \bar{u})_{H H+\alpha L+L \alpha}+D \tilde{\Omega}(N u, u)+D \tilde{\Omega}(u, N u) .
\end{aligned}
$$

In the normal form reduction, we didn't use the radial symmetry. We also remark that this reduction doesn't destroy the symmetry.

\section{Strichartz estimates And nonlinear estimates}

In this section, we introduce the Strichartz norm we need. Because of the quadratic term, our spaces rely heavily on the radial symmetry. The homogeneous Besov and the inhomogeneous Sobolev spaces on $\mathbb{R}^{3}$ are defined respectively by

$$
\|\varphi\|_{\dot{B}_{p, q}^{s}}=\left\|2^{k s} P_{k} \varphi\right\|_{\ell q\left(\mathbb{Z} ; L^{p}\left(\mathbb{R}^{3}\right)\right)}, \quad\|\varphi\|_{H_{p}^{s}}=\left\|\langle D\rangle^{s} \varphi\right\|_{L^{p}\left(\mathbb{R}^{3}\right)} .
$$

The $L^{2}$-Sobolev spaces are denoted by $\dot{H}^{s}=\dot{B}_{2,2}^{s}, H^{s}=H_{2}^{s}$. For $u$ and $N$, we use the radial-improved Strichartz norms

$$
\begin{aligned}
& u \in\langle D\rangle^{-1}\left(L_{t}^{\infty} L_{x}^{2} \cap L^{2} \dot{B}_{q(\varepsilon), 2}^{1 / 4+\varepsilon}\right), \\
& N \in L_{t}^{\infty} L_{x}^{2} \cap L_{t}^{2} \dot{B}_{q(-\varepsilon), 2}^{-1 / 4-\varepsilon},
\end{aligned}
$$

for fixed $0<\varepsilon \ll 1$, where $q(\cdot)$ is defined by

$$
\frac{1}{q(\varepsilon)}=\frac{1}{4}+\frac{\varepsilon}{3},
$$

such that we have the Sobolev embedding

$$
\dot{B}_{q\left(s_{1}\right), \rho_{1}}^{s_{1}} \subset \dot{B}_{q\left(s_{2}\right), \rho_{2}}^{s_{2}} \quad\left(s_{1} \geq s_{2}, \rho_{1} \geq \rho_{2}\right) .
$$

In particular, we have

$$
\dot{H}^{1}=\dot{B}_{q(3 / 4), 2}^{1} \subset \dot{B}_{q(\varepsilon), 2}^{1 / 4+\varepsilon} \subset \dot{B}_{q(-\varepsilon), 2}^{1 / 4-\varepsilon} \subset L^{6} .
$$

From now on, the third exponent of the Besov space will be fixed to 2 and so omitted. The condition $0<\varepsilon \ll 1$ ensures that

$$
\frac{10}{3}<q(\varepsilon)<4<q(-\varepsilon)<\infty
$$

such that the norms in (3.2) are Strichartz-admissible for radial solutions. The Strichartz estimates that we will use are given in the following lemma, and refer to [6] for their proof as well as some preceding results.

Lemma 3.1. Assume $\phi(x), f(t, x)$ are spatially radially symmetric in $\mathbb{R}^{3}$. Then (1) if $(q, r),(\tilde{q}, \tilde{r})$ both satisfy the Schrödinger-admissible condition:

$$
2 \leq q \leq \infty, \frac{2}{q}+\frac{5}{r}<\frac{5}{2} ; \text { or }(q, r)=(\infty, 2)
$$


and $\tilde{q}>2$, then

$$
\begin{aligned}
& \|S(t) \phi\|_{L_{t}^{q} \dot{B}_{r, 2}^{\frac{2}{q}+\frac{3}{r}-\frac{3}{2}}} \lesssim\|\phi\|_{L^{2}}, \\
& \left\|\int_{0}^{t} S(t-s) f(s) d s\right\|_{L_{t}^{q} \dot{B}_{r, 2}^{\frac{2}{q}+\frac{3}{r}-\frac{3}{2}}} \lesssim\|f\|_{L_{t}^{\tilde{q}^{\prime}} \dot{B}_{\tilde{r}^{\prime}, 2}^{\frac{3}{2}-\frac{2}{q}-\frac{3}{r}}} .
\end{aligned}
$$

(2) if $(q, r),(\tilde{q}, \tilde{r})$ both satisfy the wave-admissible condition:

$$
2 \leq q \leq \infty, \frac{1}{q}+\frac{2}{r}<1 ; \text { or }(q, r)=(\infty, 2)
$$

and $\tilde{q}>2$, then

$$
\begin{aligned}
& \left\|W_{\alpha}(t) \phi\right\|_{L_{t}^{q} \dot{B}_{r, 2}^{\frac{1}{q}+\frac{3}{r}-\frac{3}{2}}} \lesssim\|\phi\|_{L^{2}} \\
& \left\|\int_{0}^{t} W_{\alpha}(t-s) f(s) d s\right\|_{L_{t}^{q} \dot{B}_{r, 2}^{\frac{1}{q}+\frac{3}{r}-\frac{3}{2}}} \lesssim\|f\|_{L_{t}^{\tilde{q}^{\prime}} \dot{B}_{\tilde{r}^{\prime}, 2}^{\frac{3}{2}-\frac{1}{q}-\frac{3}{r}}} .
\end{aligned}
$$

We intend to apply this lemma to the integral equations. Then in order to close the argument, we need to do some nonlinear estimates.

3.1. Bilinear terms. The above Strichartz norms neatly fit in the bilinear terms on the right, which are partially resonant. Indeed we have

Lemma 3.2. (1) For any $N$ and $u$, the following estimates hold

$$
\begin{aligned}
&\left\|(N u)_{L H}\right\|_{L_{t}^{1} H_{x}^{1}} \lesssim\|N\|_{L_{t}^{2} \dot{B}_{q(-\varepsilon)}^{-1 / 4-\varepsilon}}\|\langle D\rangle u\|_{L_{t}^{2} \dot{B}_{q(\varepsilon)}^{1 / 4+\varepsilon}}, \\
&\left\|(N u)_{H H}\right\|_{L_{t}^{1} H_{x}^{1}} \lesssim\|N\|_{L_{t}^{2} \dot{B}_{q(-\varepsilon)}^{-1 / 4-\varepsilon}}\|\langle D\rangle u\|_{L_{t}^{2} \dot{B}_{q(\varepsilon)}^{1 / 4+\varepsilon}} .
\end{aligned}
$$

(2) If $0 \leq \theta \leq 1, \frac{1}{\tilde{q}}=\frac{1}{2}-\frac{\theta}{2}, \frac{1}{\tilde{r}}=\frac{1}{4}+\frac{\theta}{3}+\frac{\varepsilon}{3}$, then for any $N$ and $u$

$$
\left\|(N u)_{\alpha L}\right\|_{\langle D\rangle^{-1} L_{t}^{\tilde{q}^{\prime}} \dot{B}_{\bar{r}^{\prime}}^{\frac{3}{2}-\frac{2}{q}-\frac{3}{\tau}}} \lesssim\|N\|_{L_{t}^{2} \dot{B}_{q(-\varepsilon)}^{-1 / 4-\varepsilon}}\|u\|_{L_{t}^{\infty} L_{x}^{2} \cap L_{t}^{2} \dot{B}_{q(\varepsilon)}^{1 / 4+\varepsilon}} .
$$

Proof. First we prove (1). For the first inequality, it suffices to prove

$$
\left\|(N u)_{L H}\right\|_{H_{x}^{1}} \lesssim\|N\|_{\dot{B}_{q(-\varepsilon)}^{-1 / 4-\varepsilon}}\|\langle D\rangle u\|_{\dot{B}_{q(\varepsilon)}^{1 / 4+\varepsilon}} .
$$

By dyadic decomposition, we have $(N u)_{L H}=\sum_{k_{1} \leq k_{2}-5} P_{k_{1}} N P_{k_{2}} u$. Then by Hölder inequality, we get

$$
\begin{aligned}
& \left\|\langle D\rangle(N u)_{L H}\right\|_{L^{2}} \\
& \leq\left(\sum_{k_{2}}\left|\sum_{k_{1} \leq k_{2}-5}\left(2^{k_{2}}+1\right)\left\|P_{k_{1}} N\right\|_{q(-\varepsilon)}\left\|P_{k_{2}} u\right\|_{q(\varepsilon)}\right|^{2}\right)^{1 / 2} \\
& \lesssim\left(\sum_{k_{2}}\left|\sum_{k_{1} \leq k_{2}-5} 2^{k_{1}\left(\frac{1}{4}+\varepsilon\right)} 2^{k_{1}\left(-\frac{1}{4}-\varepsilon\right)}\left\|P_{k_{1}} N\right\|_{q(-\varepsilon)}\left(1+2^{k_{2}}\right)\left\|P_{k_{2}} u\right\|_{q(\varepsilon)}\right|^{2}\right)^{1 / 2} \\
& \lesssim\|N\|_{\dot{B}_{q(-\varepsilon)}^{-1 / 4-\varepsilon}}\|\langle D\rangle u\|_{\dot{B}_{q(\varepsilon)}^{1 / 4+\varepsilon} .}
\end{aligned}
$$


Similarly, for the second one, we get

$$
\begin{aligned}
\left\|\langle D\rangle(N u)_{H H}\right\|_{L^{2}} & \leq \sum_{\left|k_{1}-k_{2}\right| \lesssim 4}\left\|P_{k_{1}} N\right\|_{q(-\varepsilon)}\left(1+2^{k_{2}}\right)\left\|P_{k_{2}} u\right\|_{q(\varepsilon)} \\
& \lesssim\|N\|_{\dot{B}_{q(-\varepsilon)}^{-1 / 4-\varepsilon}}\|\langle D\rangle u\|_{\dot{B}_{q(\varepsilon)}^{1 / 4+\varepsilon}}
\end{aligned}
$$

For (2), as $(N u)_{\alpha L}$ is supported in a fixed band $|\xi| \sim \alpha$, then we have

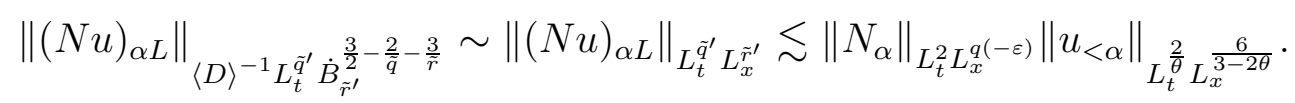

Thus (2) follows by Sobolev embedding and interpolation.

Remark 2. In application, we will use Lemma 3.2 (2) by fixing a $\theta_{0}$ such that $0<\theta_{0}<\frac{3}{8}-\frac{5 \varepsilon}{2}$. By this choice, $(\tilde{q}, \tilde{r})$ is admissible to apply Lemma 3.1 (1).

Lemma 3.3. (1) For any $u$, we have

$$
\left\|D(u \bar{u})_{H H}\right\|_{L_{t}^{1} L_{x}^{2}} \lesssim\|u\|_{L_{t}^{2} \dot{B}_{q(-\varepsilon)}^{1 / 4-\varepsilon}}\|\langle D\rangle u\|_{L_{t}^{2} \dot{B}_{q(\varepsilon)}^{1 / 4+\varepsilon}}
$$

(2) If $0 \leq \theta \leq 1, \frac{1}{\tilde{q}}=\frac{1}{2}-\frac{\theta}{2}, \frac{1}{\tilde{r}}=\frac{1}{4}+\frac{\theta}{3}-\frac{\varepsilon}{3}$, then

$$
\left\|D(u \bar{u})_{\alpha L+L \alpha}\right\|_{L_{t}^{\tilde{q}^{\prime}} \dot{B}_{\tilde{r}^{\prime}}^{\frac{3}{2}-\frac{1}{q}-\frac{3}{r}}} \lesssim\|\langle D\rangle u\|_{L_{t}^{\infty} L_{x}^{2} \cap L_{t}^{2} \dot{B}_{q(\varepsilon)}^{1 / 4+\varepsilon}}^{2} .
$$

Proof. The proof is similar to that of previous lemma. We omit the details.

Remark 3. In application, we will use Lemma 3.3 (2) by fixing a $\theta_{0}$ such that $0<\theta_{0}<4 \varepsilon$. By this choice, $(\tilde{q}, \tilde{r})$ is admissible to apply Lemma 3.1 (2).

3.2. Boundary terms. Next, we estimate the boundary terms.

Lemma 3.4. For any $N_{0}$ and $u_{0}$, we have

$$
\left\|\Omega\left(N_{0}, u_{0}\right)\right\|_{H_{x}^{1}} \lesssim\left\|N_{0}\right\|_{L_{x}^{2}}\left\|u_{0}\right\|_{H_{x}^{1}},\left\|D \tilde{\Omega}\left(u_{0}, u_{0}\right)\right\|_{L_{x}^{2}} \lesssim\left\|u_{0}\right\|_{H_{x}^{1}}\left\|u_{0}\right\|_{H_{x}^{1}} .
$$

As a consequence, for any $N$ and $u$

$$
\|\Omega(N, u)\|_{L_{t}^{\infty} H_{x}^{1}} \lesssim\|N\|_{L_{t}^{\infty} L_{x}^{2}}\|u\|_{L_{t}^{\infty} H_{x}^{1}},\|D \tilde{\Omega}(u, u)\|_{L_{t}^{\infty} L_{x}^{2}} \lesssim\|u\|_{L_{t}^{\infty} H_{x}^{1}}\|u\|_{L_{t}^{\infty} H_{x}^{1}}
$$

Proof. We only prove $\left\|\Omega\left(N_{0}, u_{0}\right)\right\|_{H_{x}^{1}} \lesssim\left\|N_{0}\right\|_{L_{x}^{2}}\left\|u_{0}\right\|_{H_{x}^{1}}$, since the others are similar. By Plancherel, Young and Schwarz we have

$$
\begin{aligned}
\left\|\Omega\left(N_{0}, u_{0}\right)\right\|_{H_{x}^{1}} & \lesssim\left\|\int_{|\xi-\eta| \gg|\eta|}|\eta|^{-1}\left|\hat{N}_{0}(\xi-\eta)\right| \cdot\left|\hat{u}_{0}(\eta)\right| d \eta\right\|_{L_{\xi}^{2}} \\
& \leq\left\|\hat{N}_{0}\right\|_{L_{\xi}^{2}}\left\||\xi|^{-1} \hat{u}_{0}\right\|_{L_{\xi}^{1}} \leq\left\|\hat{N}_{0}\right\|_{L_{\xi}^{2}}\left\||\xi|^{-1}\langle\xi\rangle^{-1}\right\|_{L_{\xi}^{2}}\left\|\langle\xi\rangle \hat{u}_{0}\right\|_{L_{\xi}^{2}} \\
& \lesssim\left\|N_{0}\right\|_{L_{x}^{2}}\left\|u_{0}\right\|_{H_{x}^{1}} .
\end{aligned}
$$

To handle the other component, we will need the following Coifman-Meyer type bilinear multiplier estimates:

Lemma 3.5. Let $T_{m}$ be the bilinear operator on $\mathbb{R}^{n}(n \geq 1)$

$$
T_{m}(f, g)(x)=\int_{\mathbb{R}^{2 n}} m(\xi, \eta) \hat{f}(\xi) \hat{g}(\eta) e^{i x(\xi+\eta)} d \xi d \eta
$$


Assume $m$ is bounded and satisfies for all $\alpha, \beta$

$$
\left|\partial_{\xi}^{\alpha} \partial_{\eta}^{\beta} m(\xi, \eta)\right| \leq C_{\alpha \beta}|\xi|^{-|\alpha|}|\eta|^{-|\beta|} .
$$

If $1 \leq p, q, r \leq \infty$ and $1 / r=1 / p+1 / q$, then for any $k_{1}, k_{2} \in \mathbb{Z}$ we have

$$
\left\|T_{m}\left(P_{k_{1}} f, P_{k_{2}} g\right)\right\|_{L^{r}} \leq C\|f\|_{L^{p}}\|g\|_{L^{q}} .
$$

Proof. We can write

$$
T_{m}\left(P_{k_{1}} f, P_{k_{2}} g\right)(x)=\int K\left(x-y, x-y^{\prime}\right) f(y) g\left(y^{\prime}\right) d y d y^{\prime}
$$

where the kernel

$$
K(x, y)=\int m(\xi, \eta) \chi_{k_{1}}(\xi) \chi_{k_{2}}(\eta) e^{i x \xi+i y \eta} d \xi d \eta .
$$

From the assumption on $m$, and integration by parts, we get a pointwise bound

$$
|K(x, y)| \lesssim 2^{k_{1} n}\left(1+\left|2^{k_{1}} x\right|\right)^{-n-1} 2^{k_{2} n}\left(1+\left|2^{k_{2}} y\right|\right)^{-n-1} .
$$

Thus the lemma follows from Hölder's inequality and Minkowski's inequality.

Lemma 3.6. For any $N$ and $u$ we have

$$
\begin{gathered}
\|\langle D\rangle \Omega(N, u)\|_{L_{t}^{2} \dot{B}_{q(\varepsilon)}^{1 / 4+\varepsilon}} \lesssim\|N\|_{L_{t}^{\infty} L_{x}^{2}}\|u\|_{L_{t}^{2} H_{6}^{1}}, \\
\|D \tilde{\Omega}(u, u)\|_{L_{t}^{2} \dot{B}_{q(-\varepsilon)}^{-1 / 4-\varepsilon}} \lesssim\|u\|_{L_{t}^{2} L_{x}^{6}}\|u\|_{L_{t}^{\infty} L_{x}^{2}} .
\end{gathered}
$$

Proof. For the first inequality, it suffices to prove

$$
\|\langle D\rangle \Omega(N, u)\|_{\dot{B}_{q(\varepsilon)}^{1 / 4+\varepsilon}} \lesssim\|N\|_{L_{x}^{2}}\|u\|_{H_{6}^{1}}
$$

By Sobolev embedding, we get $\|\langle D\rangle \Omega(N, u)\|_{\dot{B}_{q(\varepsilon)}^{1 / 4+\varepsilon}} \lesssim\|D\langle D\rangle \Omega(N, u)\|_{L^{2}}$. It is easy to see that $D\langle D\rangle \Omega(N, u)$ is a bilinear multiplier with the symbol

$$
m(\xi, \eta)=\frac{|\xi+\eta|\langle\xi+\eta\rangle \sum_{2^{k} \nsim \alpha} \chi_{\leq k-5}(\eta) \chi_{k}(\xi)}{-|\xi+\eta|^{2}+\alpha|\eta|+|\xi|^{2}},
$$

and $m$ satisfies the condition in Lemma 3.5. Thus applying dyadic decomposition and Lemma 3.5, we get

$$
\begin{aligned}
\|D\langle D\rangle \Omega(N, u)\|_{L^{2}} & \lesssim\left(\sum_{k_{2}}\left\|\sum_{k_{1} \leq k_{2}-5} D\langle D\rangle \Omega\left(P_{k_{2}} N, P_{k_{1}} u\right)\right\|_{2}^{2}\right)^{1 / 2} \\
& \lesssim\left(\sum_{k_{2}}\left(\sum_{k_{1} \leq k_{2}-5}\left\|P_{k_{2}} N\right\|_{L^{2}}\left\|P_{k_{1}} u\right\|_{\infty}\right)^{2}\right)^{1 / 2} \lesssim\|N\|_{L_{x}^{2}}\|u\|_{H_{6}^{1}}
\end{aligned}
$$

where we used $\sum_{k_{1} \leq k_{2}-5}\left\|P_{k_{1}} u\right\|_{\infty} \lesssim \sum_{k_{1} \leq k_{2}-5} 2^{k_{1} / 2}\left\|P_{k_{1}} u\right\|_{6} \leq\|u\|_{H_{6}^{1}}$.

Similarly, for the second inequality, by Sobolev embedding we get

$$
\|D \tilde{\Omega}(u, u)\|_{\dot{B}_{q(-\varepsilon)}^{-1 / 4-\varepsilon}} \lesssim\left\|D^{3 / 2} \tilde{\Omega}(u, u)\right\|_{L^{2}}
$$


and $D^{2} \tilde{\Omega}$ behaves similarly to $D\langle D\rangle \Omega$. Then applying dyadic decomposition and Lemma 3.5, we get

$$
\begin{aligned}
\left\|D^{3 / 2} \tilde{\Omega}(u, u)\right\|_{L^{2}} & \lesssim\left(\sum_{k_{2}}\left\|\sum_{k_{1} \leq k_{2}-5} D^{3 / 2} \tilde{\Omega}\left(P_{k_{2}} u, P_{k_{1}} u\right)\right\|_{2}^{2}\right)^{1 / 2} \\
& \lesssim\left(\sum_{k_{2}}\left(\sum_{k_{1} \leq k_{2}-5} 2^{-k_{2} / 2}\left\|P_{k_{2}} u\right\|_{L^{2}}\left\|P_{k_{1}} u\right\|_{\infty}\right)^{2}\right)^{1 / 2} \lesssim\|u\|_{L_{x}^{2}}\|u\|_{L_{x}^{6}}
\end{aligned}
$$

Thus we finish the proof of the lemma.

3.3. Cubic terms. Finally, we deal with the cubic terms.

Lemma 3.7. For any $N$ and $u$ we have

$$
\begin{aligned}
\left\|\Omega\left(D|u|^{2}, u\right)\right\|_{L_{t}^{1} H_{x}^{1}} & \lesssim\|\langle D\rangle u\|_{L_{t}^{2} L_{x}^{6}}^{2}\|u\|_{L_{t}^{\infty} L_{x}^{2}}, \\
\|\langle D\rangle \Omega(N, N u)\|_{L_{t}^{2} \dot{B}_{6 / 5,2}^{0}} & \lesssim\|\langle D\rangle u\|_{L_{t}^{2} L_{x}^{6}}\|N\|_{L_{t}^{\infty} L_{x}^{2}}^{2}, \\
\|D \tilde{\Omega}(N u, u)\|_{L_{t}^{1} L_{x}^{2}} & \lesssim\|\langle D\rangle u\|_{L_{t}^{2} L_{x}^{6}}^{2}\|N\|_{L_{t}^{\infty} L_{x}^{2}}
\end{aligned}
$$

Proof. As in the proof of previous lemma, applying dyadic decomposition and Lemma 3.5, we get

$$
\begin{aligned}
\left\|\Omega\left(D|u|^{2}, u\right)\right\|_{H_{x}^{1}} & \lesssim\left(\sum_{k_{2}}\left\|\sum_{k_{1} \leq k_{2}-5}\langle D\rangle \Omega\left(P_{k_{2}} D|u|^{2}, P_{k_{1}} u\right)\right\|_{2}^{2}\right)^{1 / 2} \\
& \lesssim\left(\sum_{k_{2}}\left(\sum_{k_{1} \leq k_{2}-5}\left\|P_{k_{2}}|u|^{2}\right\|_{L^{2}}\left\|P_{k_{1}} u\right\|_{L^{\infty}}\right)^{2}\right)^{1 / 2} \lesssim\|\langle D\rangle u\|_{L_{x}^{6}}^{2}\|u\|_{L_{x}^{2}} .
\end{aligned}
$$

Similarly, for the second inequality, we have

$$
\begin{aligned}
\|\langle D\rangle \Omega(N, N u)\|_{\dot{B}_{6 / 5}^{0}} & \lesssim\left(\sum_{k_{2}}\left\|\sum_{k_{1} \leq k_{2}-5}\langle D\rangle \Omega\left(P_{k_{2}} N, P_{k_{1}}(N u)\right)\right\|_{6 / 5}^{2}\right)^{1 / 2} \\
& \lesssim\left(\sum_{k_{2}}\left(\sum_{k_{1} \leq k_{2}-5} 2^{-k_{2}}\left\|P_{k_{2}} N\right\|_{L^{2}}\left\|P_{k_{1}}(N u)\right\|_{L_{x}^{3}}\right)^{2}\right)^{1 / 2} \\
& \lesssim\left(\sum_{k_{2}}\left(\sum_{k_{1} \leq k_{2}-5} 2^{-k_{2}+k_{1}}\left\|P_{k_{2}} N\right\|_{L^{2}}\|N\|_{L_{x}^{2}}\|u\|_{L_{x}^{6}}\right)^{2}\right)^{1 / 2} \\
& \lesssim\|u\|_{L_{x}^{6}}\|N\|_{L_{x}^{2}}^{2},
\end{aligned}
$$

and for the last inequality, we have

$$
\begin{aligned}
\|D \tilde{\Omega}(N u, u)\|_{L_{x}^{2}} & \lesssim\left(\sum_{k_{2}}\left\|\sum_{k_{1} \leq k_{2}-5} 2^{k_{2}} \widetilde{\Omega}\left(P_{k_{2}}(N u), P_{k_{1}} u\right)\right\|_{2}^{2}\right)^{1 / 2} \\
& \lesssim\left(\sum_{k_{2}}\left(\sum_{k_{1} \leq k_{2}-5}\left\langle 2^{k_{2}}\right\rangle^{-1}\left\|P_{k_{2}}(N u)\right\|_{L^{2}}\left\|P_{k_{1}} u\right\|_{L^{\infty}}\right)^{2}\right)^{1 / 2} \\
& \lesssim\|\langle D\rangle u\|_{L_{x}^{6}}^{2}\|N\|_{L_{x}^{2}}
\end{aligned}
$$

Using Hölder in $t$, we obtain the claimed inequalities. 


\section{Proof of Theorem 1.1}

Now we are ready to prove Theorem 1.1. The main ingredients are the estimates obtained in the previous section. For any $\left(u_{0}, n_{0}, n_{1}\right) \in H_{r}^{1}\left(\mathbb{R}^{3}\right) \times L_{r}^{2}\left(\mathbb{R}^{3}\right) \times \dot{H}_{r}^{-1}\left(\mathbb{R}^{3}\right)$, we define an operator $\Phi_{u_{0}, n_{0}, n_{1}}(u, N)$ by the right-hand side of (2.13)-(2.14). Our resolution space is

$$
X_{\eta}=\left\{(u, N):\|(u, N)\|_{X}=\|\langle D\rangle u\|_{L_{t}^{\infty} L_{x}^{2} \cap L^{2} \dot{B}_{q(\varepsilon), 2}^{1 / 4+\varepsilon}}+\|N\|_{L_{t}^{\infty} L_{x}^{2} \cap L_{t}^{2} \dot{B}_{q(-\varepsilon), 2}^{-1 / 4-\varepsilon}} \leq \eta\right\}
$$

endowed with the norm metric $\|\cdot\|_{X}$.

We will show that $\Phi_{u_{0}, n_{0}, n_{1}}: X_{\eta} \rightarrow X_{\eta}$ is a contraction mapping, provided that $\eta \ll 1$ and $\left(u_{0}, n_{0}, n_{1}\right)$ are sufficiently small. By the estimates in the previous section, we have for any $(u, N) \in X_{\eta}$

$$
\begin{aligned}
\left\|\Phi_{u_{0}, n_{0}, n_{1}}(u, N)\right\|_{X} & \lesssim\left\|u_{0}\right\|_{H^{1}}+\left\|n_{0}\right\|_{L^{2}}+\left\|n_{1}\right\|_{\dot{H}^{-1}}+\left(\left\|u_{0}\right\|_{H^{1}}+\left\|n_{0}\right\|_{L^{2}}+\left\|n_{1}\right\|_{\dot{H}^{-1}}\right)^{2} \\
& +\|(u, N)\|_{X}^{2}+\|(u, N)\|_{X}^{3} \leq \eta
\end{aligned}
$$

if $\varepsilon_{0}=\left\|u_{0}\right\|_{H^{1}}+\left\|n_{0}\right\|_{L^{2}}+\left\|n_{1}\right\|_{\dot{H}^{-1}} \ll 1$, and we set $\eta=C \varepsilon_{0}$. Similarly, we can prove $\Phi_{u_{0}, n_{0}, n_{1}}: X_{\eta} \rightarrow X_{\eta}$ is a contraction mapping. Our estimates are time global, therefore Theorem 1.1 follows immediately. The quadratic terms without time integral tend to 0 strongly in the energy space as $|t| \rightarrow \infty$, so they do not contribute to the scattering states.

Acknowledgment. This work started when the second author was visiting the Beijing International Center for Mathematical Research. He would like to thank Professor Baoxiang Wang for his kind invitation and hospitality. Z. Guo is supported in part by NNSF of China (No. 11001003).

\section{REFERENCES}

[1] I. Bejenaru and S. Herr, Convolutions of singular measures and applications to the Zakharov system. J. Funct. Anal. 261 (2011), no. 2, 478-506.

[2] I. Bejenaru, S. Herr, J. Holmer, and D. Tataru, On the 2D Zakharov system with $L^{2}$ Schrödinger data. Nonlinearity 22 (2009), no. 5, 1063-1089.

[3] J. Bourgain and J. Colliander, On wellposedness of the Zakharov system. Internat. Math. Res. Notices 1996, no. 11, 515-546.

[4] J. Ginibre and G. Velo, Scattering theory for the Zakharov system. Hokkaido Math. J. 35 (2006), no. 4, 865-892.

[5] J. Ginibre, Y. Tsutsumi and G. Velo, On the Cauchy problem for the Zakharov system. J. Funct. Anal. 151 (1997), no. 2, 384-436.

[6] Z. Guo and Y. Wang, Improved Strichartz estimates for a class of dispersive equations in the radial case and their applications to nonlinear Schrödinger and wave equation. arXiv:1007.4299.

[7] C. Kenig, G. Ponce and L. Vega, On the Zakharov and Zakharov-Schulman systems. J. Funct. Anal. 127 (1995), no. 1, 204-234.

[8] N. Kishimoto, Local well-posedness for the Zakharov system on multidimensional torus. preprint, arXiv:1109.3527.

[9] N. Masmoudi and K. Nakanishi, Energy convergence for singular limits of Zakharov type systems. Invent. Math. 172 (2008), no. 3, 535-583.

[10] F. Merle, Blow-up results of virial type for Zakharov equations. Comm. Math. Phys., 175 (1996), pp. 433-455.

[11] T. Ozawa and Y. Tsutsumi, The nonlinear Schrödinger limit and the initial layer of the Zakharov equations. Differ. Integral Equ. 5 (1992) no. 4, 721-745. 
[12] T. Ozawa and Y. Tsutsumi, Global existence and asymptotic behavior of solutions for the Zakharov equations in three-dimensions space. Adv. Math. Sci. Appl. 3 (Special Issue) (1993/94) 301-334.

[13] T. Ozawa, K. Tsutaya and Y. Tsutsumi, Well-posedness in energy space for the Cauchy problem of the Klein-Gordon-Zakharov equations with different propagation speeds in three space dimensions. Math. Ann. 313 (1999) no. 1, 127-140.

[14] S. Schochet and M. Weinstein, The nonlinear Schrödinger limit of the Zakharov equations governing Langmuir turbulence. Commun. Math. Phys. 106 (1986), no.4, 569-580.

[15] J. Shatah, Normal forms and quadratic nonlinear Klein-Gordon equations. Comm. Pure Appl. Math. 38 (1985), no. 5, 685-696.

[16] A. Shimomura, Scattering theory for Zakharov equations in three-dimensional space with large data. Commun. Contemp. Math. 6 (2004), no. 6, 881-899.

[17] H. Takaoka, Well-posedness for the Zakharov system with the periodic boundary condition. Differential Integral Equations 12 (1999), no. 6, 789-810.

[18] V. E. Zakharov, Collapse of Langmuir waves. Sov. Phys. JETP 35 (1972), 908-914.

${ }^{1}$ Lmam, School of Mathematical Sciences, Peking University, Beijing 100871, CHINA

${ }^{2}$ Beijing International Center for Mathematical Research, Beijing 100871, China

E-mail address: zihuaguo@math.pku.edu.cn

${ }^{3}$ Department of Mathematics, Kyoto University, Kyoto 606-8502, Japan

E-mail address: n-kenji@math.kyoto-u.ac.jp 